\title{
Historical Terminology, Classifications, and Present Definition of DCM
}

\author{
Marco Merlo, Chiara Daneluzzi, Luisa Mestroni, \\ Antonio Cannatà, and Gianfranco Sinagra
}

\section{Abbreviations and Acronyms}

$\begin{array}{ll}\text { ARVC } & \text { Arrhythmogenic right ventricular cardiomyopathy } \\ \text { CMPs } & \text { Cardiomyopathies } \\ \text { CMR } & \text { Cardiac magnetic resonance } \\ \text { DCM } & \text { Dilated cardiomyopathy } \\ \text { EMB } & \text { Endomyocardial biopsy } \\ \text { ESC } & \text { European Society of Cardiology } \\ \text { HF } & \text { Heart failure } \\ \text { HNDC } & \text { Hypokinetic non-dilated cardiomyopathy } \\ \text { ICD } & \text { Implantable cardioverter defibrillator } \\ \text { LMNA/C } & \text { Lamin A/C } \\ \text { LV } & \text { Left ventricular } \\ \text { LVRR } & \text { Left ventricular reverse remodeling }\end{array}$

\footnotetext{
M. Merlo $(\bowtie) \cdot$ G. Sinagra

Cardiovascular Department, Azienda Sanitaria Universitaria Integrata, Trieste, Italy

e-mail: gianfranco.sinagra@asuits.sanita.fvg.it

C. Daneluzzi · A. Cannatà

Cardiovascular Department, Azienda Sanitaria Universitaria Integrata, University of Trieste (ASUITS), Trieste, Italy
}

\section{Mestroni}

Cardiovascular Institute and Adult Medical Genetics Program, University of Colorado

Anschutz Medical Campus, Aurora, CO, USA

e-mail: luisa.mestroni@ucdenver.edu 


\subsection{Dilated Cardiomyopathies: The Classification Pathway}

Cardiomyopathies (CMPs) are myocardial disorders in which the heart muscle has structural and functional abnormalities in the absence of other causes sufficient to cause the disease. Until a few decades ago in medical literature, there was uncertainty and confusion about this entity. In the last years, advances in pathophysiology, pathology, biomarkers, genetics and molecular medicine, echocardiography, and cardiac magnetic resonance have brought light in the darkness.

Since 1956 several definitions of CMPs have been adopted using terms as "inflammatory," "non-coronary," "myocardial disorders of unknown etiology" [1]. Classifications tried to make order in the complexity and, historically, were mainly based on phenotype [2, 3] missing multiple other aspects. In 2006 the American Heart Association proposed the definition of CMPs as follows: "cardiomyopathies are a heterogeneous group of diseases of the myocardium associated with mechanical and/or electrical dysfunction that usually (but not invariably) exhibit inappropriate ventricular hypertrophy or dilatation and are due to a variety of causes that frequently are genetic. Cardiomyopathies either are confined to the heart or are part of generalized systemic disorders, often leading to cardiovascular death or progressive heart failure (HF) related disability" [4]. This classification is based on etiology, distinguishing CMP in genetic, acquired, and mixed, and splits CMPs into two groups, primary or secondary, as they involve predominately the heart or as a part of systemic disease. Brugada syndrome, long QT syndromes, short QT syndromes, catecholaminergic ventricular polymorphic tachycardia, and Asian sudden unexplained nocturnal deaths are put separately, but for the first time, channelopathies were mentioned in the classification of genetic cardiomyopathies.

Two years later the European Society of Cardiology (ESC) chose a clinical and morphological classification (Fig. 1.1), reporting CMPs as "myocardial disorders in which structure and function of the myocardium are abnormal, in the absence of coronary artery disease, hypertension, valvular heart disease and congenital heart disease sufficient to cause the observed abnormality". Dilated CMP, hypertrophic CMP, restrictive CMP, and arrhythmogenic right ventricular CMP are the four main specific phenotypes that have to be subsequently subclassified in familial and nonfamilial. Actually the picture is not so simple, with heterogeneity and overlapping forms [5].

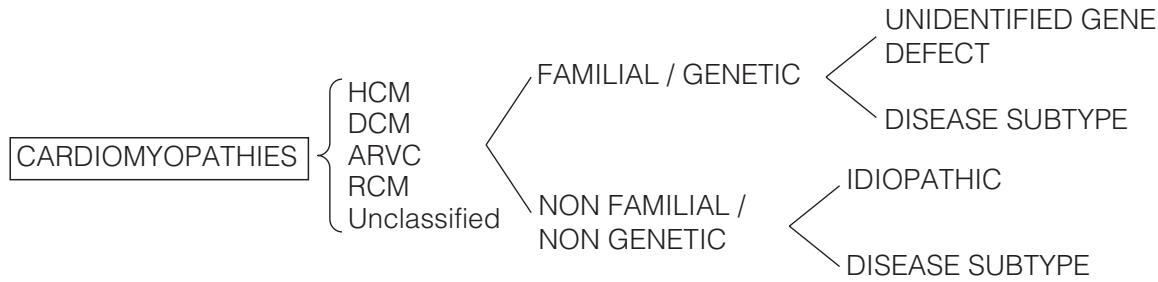

Fig. 1.1 The 2008 ESC classification of cardiomyopathies. From Elliott P. et al. European Heart Journal 2008 
The need to integrate the above multiple aspects of CMPs prompts last classification available, proposed by Arbustini et al. in 2013 and endorsed by the World Heart Federation, the MOGE(S), a morphofunctional classification, enriched with extracardiac involvement, mode of inheritance with effect of mutation on gene function, and functional status. In details $\operatorname{MOGE}(\mathrm{S})$ acronym stands for morphofunctional characteristics $(\mathrm{M})$, organ involvement $(\mathrm{O})$, genetic or familial inheritance pattern $(\mathrm{G})$, etiological information (E), and functional status (S). This system resembles the TNM classification of tumors and provides a genotype-phenotype correlation [6]. It seems to be a challenging way to describe CMPs in everyday life; however, it pushes clinicians to clarify etiology and familiar history and to have a comprehensive approach to the patients, not focusing only on the heart. Actually, even if it represents a translation link between basic science and clinical medicine, However, its use in clinical practice is rare [7].

Although major advances in knowledge as reported above, DCM is the cardiomyopathy that, between all others, still lacks of complete characterization and understanding. The term DCM encloses multiple entities, and, so far, no classification has been able to portrait it adequately.

Anyway, continuous efforts are made by researchers, and in 2016, a new statement has been published. Pinto et al. proposed a revised definition of DCM (Fig. 1.2), which tries to encompass the broad clinical features of the disease and its changes during time. They emphasize the progression of the disease from a preclinical state with no cardiac dilation through isolated ventricular dilation or arrhythmic cardiomyopathy, characterized by arrhythmogenic features as supraventricular/ventricular arrhythmias and/or conduction defects observed in myocarditis, genetic defects, and neuromuscular diseases. Furthermore, they introduce a new entity called "the hypokinetic non-dilated cardiomyopathy (HNDC)" which is the overt phase of systolic dysfunction not associated with ventricular dilation, as it happens in DCM caused by Lamin A/C defects. The final landing remains DCM [8].

Another new concept comes from the recent awareness that DCM overlaps with arrhythmogenic right ventricular cardiomyopathy (ARVC). They may share diseasecausing mutations; desmosomal gene defects are known to be mutated in DCM and

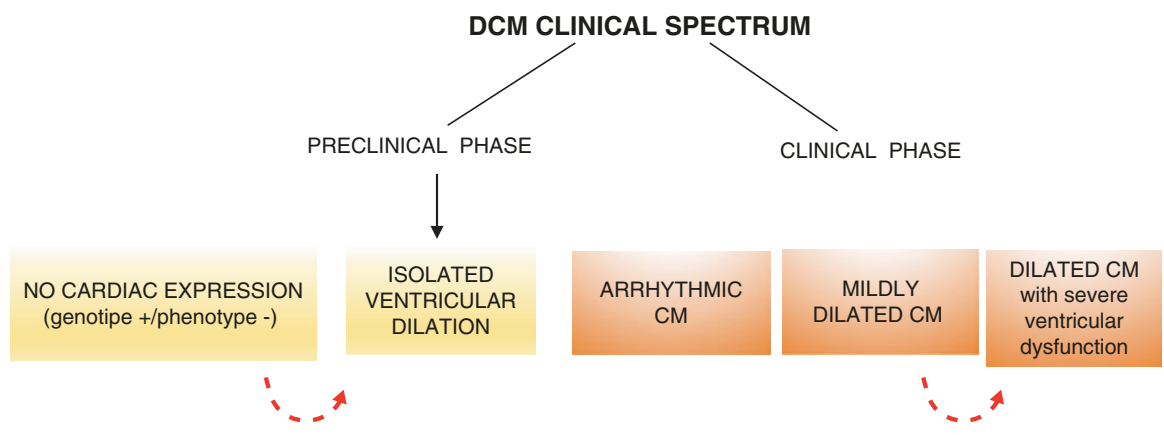

Fig. 1.2 The DCM clinical spectrum. From Pinto Y.M. et al., European Heart Journal 2016 
ARVC. Moreover, patients with ARVC can show a left ventricular involvement, and the other way around a DCM relative may demonstrate ventricular ectopy coming from the right ventricle [8].

Maybe in the next classification there will be room for this overlap form with specific gene defects.

Despite major scientific progresses in the last decades, DCM still remains the third cause of HF and the first cause of cardiac transplant worldwide, with high clinical relevance given its mortality-morbidity risk in such a young population with long life expectancy (mean age at diagnosis is 45 years) (Fig. 1.3).

Major advances have been made in DCM since the 1980s when it was considered an end-stage condition, as a cancer, with $50 \%$ of mortality at 2 years. Nowadays, the estimated free survival from death and heart transplant is approximately of $85 \%$ at 10 years [9]. This is the result of earlier diagnosis with consequent earlier beginning of evidence-based therapy, which has dramatically improved in the last 30 years with introduction of neurohormonal agent (most recent sacubitril-valsartan) and non-pharmacological therapy (implantable cardioverter defibrillator (ICD) and resynchronization therapy). Unfortunately, we are not always able to adequately
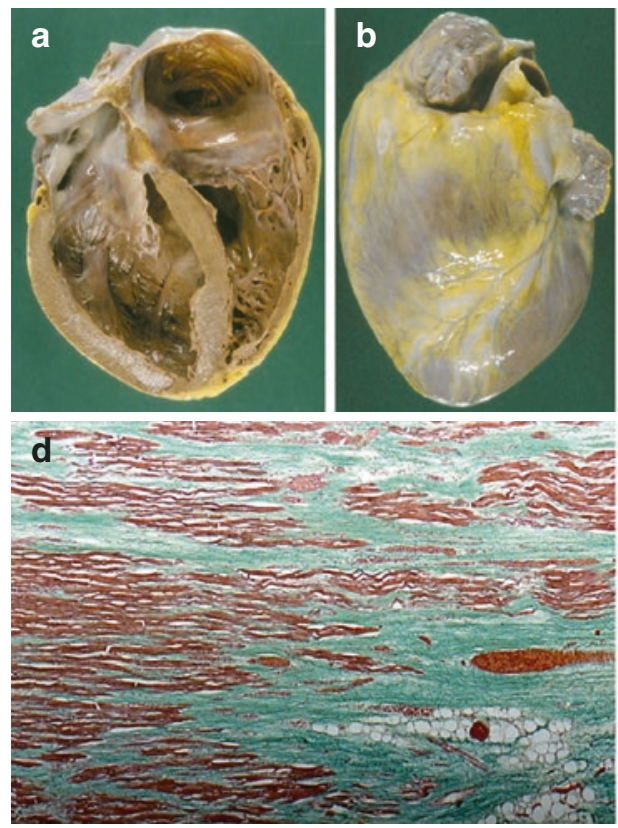
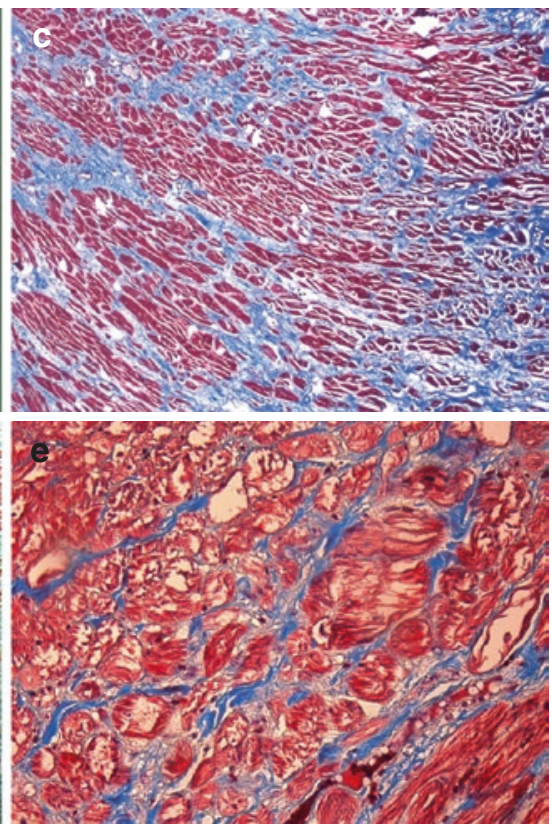

Fig. 1.3 Gross anatomy and histological specimen representative of DCM. (a, b) Gross anatomy of an explanted heart from a 26-year-old patient with DCM. (c) Azan-Mallory staining of a female patient with DCM and severe LV dysfunction; (d) histology from a patient with Duchenne's dystrophy; (e) Azan-Mallory staining from an explanted heart from a patient affected by genetically determined DCM (double mutation in desmin and potassium channels). Courtesy of Prof. Bussani, University of Trieste 
stratify the risk in this population, especially at the beginning of the disease when the adverse left ventricular remodeling is not the only adverse predictor and major arrhythmic events can happen in patients not satisfying criteria for ICD implantation. Anyway, severe mitral regurgitation, right ventricular dysfunction, and restrictive filling pattern have been recognized as predictors of adverse events as expression of advanced disease [10-12]. On the other hand, caution has to be taken to avoid early useless ICD implantation motivated only by low ejection fraction: studies have demonstrated that left ventricular reverse remodeling is a process that lasts 3-9 months after the diagnosis (to be completed in 24 months) [13]. A global evaluation comprehensive of late gadolinium enhancement and peak circumferential strain assessed by cardiac magnetic resonance (CMR) performs better than clinicalechocardiographic evaluation alone in the prediction of left ventricular reverse remodeling (LVRR) in patient recently diagnosed with DCM receiving evidencebased therapy [14].

DCM carries important ethical issues as the identification of asymptomatic carriers of gene mutations in a family, potential risk of pregnancy, and sport participation. These are common situations that the clinical cardiologist has to face with, often without specific guidelines.

Some help in the management of DCM comes from registries enrolling clinical, instrumental, and prognostic data of large cohorts of patients affected and strictly followed in the long term. In our Institution this is a common behavior, since we can extrapolate thousands of data from the Heart Muscle Disease Registry, active from 1978 [15].

\subsection{Genetic Dilated Cardiomyopathy and Etiological Classification}

Familial forms account for the at least $40 \%$ of cases, and thanks to the recent discoveries in the genetic field, clinicians have the opportunity, but also the responsibility, to provide an etiological diagnosis, stratify the risk and treat patients with the best strategy available. So, when acquired causes (e.g. hypertension, coronary artery disease, valvular heart disease, arrhythmias, etc.) have been excluded, there is a family history of DCM and there are clinical clues suggesting the diagnosis (what we used to call "red flags": deafness, blindness, muscular disorders, etc.), we recommend to perform the genetic screening [13]. Anyway, it has to be stressed that de novo mutations exist, so a negative family history doesn't rule out a genetic DCM, and that is mandatory for an appropriate patient selection in order to avoid noise, as will be explained below.

Guidelines and position papers recommend, with level $\mathrm{C}$ of evidence, genetic testing in the proband (the first or the most affected in the family, as this gives a high positive predictive value) in order to provide diagnostic/prognostic information, aid therapeutic choices, and prompt cascade screening in relatives [16]. Family screening allows an early diagnosis in a consistent number of patients, facilitating the diagnosis in non-proband DCM patients at an early stage of the disease, giving the chance to start optimal medical therapy earlier [17]. 
Genetic background of DCM is a wide and complex issue. So far, more than 50 genes encoding for cytoskeleton, sarcomeric proteins, sarcolemma, nuclear envelope ion channels, and intercellular junctions have been found to be implicated in DCM, and several other genes remain to be discovered. There is variable clinical presentation (also in the same family), incomplete penetrance, age-related penetrance, and lack of specific phenotype (meaning that the same gene mutation can cause different cardiomyopathies) [13].

However, unlike few decades ago, when cardiomyopathies were a confused matter, now we are living an historical breakthrough: from a pure phenotype classification, we are moving toward a best understanding of DCM and a more "personal" characterization of the disease, thanks to genetics [18]. In particular, there is growing evidence in the field of genotype-phenotype correlation with remarkable implications in the management of patients.

Although a strong genotype-phenotype relationship is currently accepted only for LMNA/C, recently a body of data is emerging in this field. Some rare sarcomeric variants carry poor prognosis after the age of 50, supporting the role of genetic testing in further risk stratification [19]. Furthermore, cytoskeleton Z-disk mutations are demonstrated as inversely related with LVRR. Moreover, since these proteins are not involved in beta-adrenergic activity, they are not targeted by antineurohormonal drugs limiting the therapeutic effect of the widespread molecules used in HFß management [20].

Thus, the updated approach to DCM is now comprehensive of genetic evaluation with identification of genes and their corresponding phenotypic expression, accepting that most genotype-phenotype correlation remains unknown and, to date, globally, the genetic background is not able to predict disease evolution and response to therapy.

\subsection{Future Perspectives}

As frequently happens in medicine, there are unresolved issues, which are outlined below and which will be further explained in the focused chapters of the book.

Our efforts must focus on identifying the underlying DCM cause, in order to further reduce the number of "idiopathic DCM." Progresses have been made in this field; we know that in the 1980s, almost 50\% of DCM didn't have a specific cause. Nowadays the etiologic characterization has dramatically improved so that it is possible to understand the etiologic basis of many so-called idiopathic heart muscle disease [3].

Thanks to etiology-directed management, the DCM prognosis has considerably improved and clinicians must persist in this task [21].

In patient with clinically suspected myocarditis as a possible explanation for ventricular dysfunction, there is the need to proceed with endomyocardial biopsy (EMB), with histopathology, immunohistochemistry, and molecular analysis. It has a fundamental role in identifying the underlying etiology (e.g., giant cell, eosinophilic myocarditis, sarcoidosis) which imply different treatments and prognosis. It is also the basis for safe immunosuppressive therapy, after the exclusion of viral infection [22]. 
Valuable aids in the etiologic characterization of DCM come from the recent advances in echocardiography.

An interesting tool is speckle-tracking strain analysis for assessing cardiac mechanics and segmental and global LV function. This technique allows the evaluation of myocardial deformation in all its components (i.e., longitudinal and circumferential shortening and radial thickening). All parameters may be reduced in DCM, beginning in the preclinical phase and allowing an early identification of disease [23].

Another essential tool is cardiovascular magnetic resonance (CMR). It provides additional prognostic information as it is the gold standard technique for biventricular morphological and functional evaluation and tissue characterization [24].

It is frequently adopted in the setting of myocarditis in stable patients or after EMB in life-threatening presentations, according to Lake Louise criteria [22].

A step toward a comprehensive DCM classification and an attempt to reconcile clinic with genetic in the complexity of the disease is genotype-phenotype correlation, with its prognostic implication in clinical practice. A clear example of this relation is the LMNA/C, but other gene defects are emerging, such as Filamin $\mathrm{C}$ [25]. It is possible that in the future genetic cluster classification will be completed studying every gene mutation, thanks to whole-genome sequencing, taking care of the patient instead of the disease.

Our efforts are focused on a personalized medicine approach including technologies at the services of each patient maybe with genic therapy or specific antiinflammatory therapy targeted to the specific etiology.

\section{References}

1. Arbustini E, Narula N, Tavazzi L, et al. The MOGE(S) classification of cardiomyopathy for clinicians. J Am Coll Cardiol. 2014;64:304-18. https://doi.org/10.1016/j.jacc.2014.05.027.

2. Heartjf B. Report of the WHO/ISFC task force on the definition and classification of cardiomyopathies. Br Heart J. 1980;44:672-3. https://doi.org/10.1136/hrt.44.6.672.

3. Richardson P, McKenna W, Bristow M, et al. Report of the 1995 World Health Organization/ International Society and Federation of Cardiology Task Force on the definition and classification of cardiomyopathies. Circulation. 1996;93:841-2. https://doi.org/10.1161/01. CIR.93.5.841.

4. Maron BJ, Towbin JA, Thiene G, et al. Contemporary definitions and classification of the cardiomyopathies: an American Heart Association scientific statement from the Council on Clinical Cardiology, Heart Failure and Transplantation Committee. Circulation. 2006;113:1807-16. https://doi.org/10.1161/CIRCULATIONAHA.106.174287.

5. Elliott $\mathrm{P}$, Andersson B, Arbustini E, et al. Classification of the cardiomyopathies: a position statement from the European Society of Cardiology Working Group on Myocardial and Pericardial Diseases. Eur Heart J. 2008;29:270-6. https://doi.org/10.1093/eurheartj/ehm342.

6. Şahan E, Şahan S, Karamanlıoğlu M, et al. The MOGE(S) classification. Herz. 2016;41:503-6. https://doi.org/10.1007/s00059-015-4394-0.

7. Fedele F, Severino P, Calcagno S, Mancone M. Heart failure: TNM-like classification. J Am Coll Cardiol. 2014;63:1959-60.

8. Pinto YM, Elliott PM, Arbustini E, et al. Proposal for a revised definition of dilated cardiomyopathy, hypokinetic non-dilated cardiomyopathy, and its implications for clinical practice: 
a position statement of the ESC Working Group on Myocardial and Pericardial Diseases. Eur Heart J. 2016;37:1850-8. https://doi.org/10.1093/eurheartj/ehv727.

9. Merlo M, Gigli M, Poli S, et al. La cardiomiopatia dilatativa come malattia dinamica: storia naturale, rimodellamento inverso e stratificazione prognostica. G Ital Cardiol. 2016;17:15-23.

10. Merlo M, Pyxaras SA, Pinamonti B, et al. Prevalence and prognostic significance of left ventricular reverse remodeling in dilated cardiomyopathy receiving tailored medical treatment. $\mathrm{J}$ Am Coll Cardiol. 2011;57:1468-76. https://doi.org/10.1016/j.jacc.2010.11.030.

11. Stolfo D, Merlo M, Pinamonti B, et al. Early improvement of functional mitral regurgitation in patients with idiopathic dilated cardiomyopathy. Am J Cardiol. 2015;115:1137-43. https://doi. org/10.1016/j.amjcard.2015.01.549.

12. Merlo M, Stolfo D, Anzini M, et al. Persistent recovery of normal left ventricular function and dimension in idiopathic dilated cardiomyopathy during long-term follow-up: does real healing exist? J Am Heart Assoc. 2015;4:e001504. https://doi.org/10.1161/JAHA.114.000570.

13. Merlo M, Cannatà A, Gobbo M, et al. Evolving concepts in dilated cardiomyopathy. Eur J Heart Fail. 2017;20(2):228-39. https://doi.org/10.1002/ejhf.1103.

14. Merlo M, Masè M, Vitrella G, et al. Comprehensive structural and functional assessment for prediction of left ventricular reverse remodeling in non-ischemic cardiomyopathy. J Am Coll Cardiol. 2018;71:A877. https://doi.org/10.1016/S0735-1097(18)31418-9.

15. Mestroni L, Rocco C, Gregori D, et al. Familial dilated cardiomyopathy: evidence for genetic and phenotypic heterogeneity. Heart Muscle Disease Study Group. J Am Coll Cardiol. 1999;34:181-90.

16. Hershberger RE, Lindenfeld J, Mestroni L, et al. Genetic evaluation of cardiomyopathy-a Heart Failure Society of America practice guideline. J Card Fail. 2009;15:83-97. https://doi. org/10.1016/j.cardfail.2009.01.006.

17. Moretti M, Merlo M, Barbati G, et al. Prognostic impact of familial screening in dilated cardiomyopathy. Eur J Heart Fail. 2010;12:922-7. https://doi.org/10.1093/eurjhf/hfq093.

18. Sinagra G, Mestroni L, Camerini F, editors. Genetic cardiomyopathies: a clinical approach. Milan: Springer; 2013.

19. Merlo M, Sinagra G, Carniel E, et al. Poor prognosis of rare sarcomeric gene variants in patients with dilated cardiomyopathy. Clin Transl Sci. 2013;6:424-8. https://doi.org/10.1111/ cts.12116.

20. Dal Ferro M, Stolfo D, Altinier A, et al. Association between mutation status and left ventricular reverse remodelling in dilated cardiomyopathy. Heart. 2017;103:1704-10. https://doi. org/10.1136/heartjnl-2016-311017.

21. Merlo M, Gentile P, Naso P, Sinagra G. The natural history of dilated cardiomyopathy: how has it changed? J Cardiovasc Med. 2017;18:e161-5. https://doi.org/10.2459/ JCM.0000000000000459.

22. Caforio ALP, Pankuweit S, Arbustini E, et al. Current state of knowledge on aetiology, diagnosis, management, and therapy of myocarditis: a position statement of the European Society of Cardiology Working Group on Myocardial and Pericardial Diseases. Eur Heart J. 2013;34:2636-48. https://doi.org/10.1093/eurheartj/eht210.

23. Lang RM, Badano LP, Mor-Avi V, et al. Recommendations for cardiac chamber quantification by echocardiography in adults: an update from the American Society of Echocardiography and the European Association of Cardiovascular Imaging. Eur Heart J Cardiovasc Imaging. 2015;16:233-71. https://doi.org/10.1093/ehjci/jev014.

24. Hombach V, Merkle N, Torzewski J, et al. Electrocardiographic and cardiac magnetic resonance imaging parameters as predictors of a worse outcome in patients with idiopathic dilated cardiomyopathy. Eur Heart J. 2009;30:2011-8. https://doi.org/10.1093/eurheartj/ehp293.

25. Corrado D, Zorzi A. Filamin C: a new arrhythmogenic cardiomyopathy-causing gene? JACC Clin Electrophysiol. 2018;4:515-7. 
Open Access This chapter is licensed under the terms of the Creative Commons Attribution 4.0 International License (http://creativecommons.org/licenses/by/4.0/), which permits use, sharing, adaptation, distribution and reproduction in any medium or format, as long as you give appropriate credit to the original author(s) and the source, provide a link to the Creative Commons license and indicate if changes were made.

The images or other third party material in this chapter are included in the chapter's Creative Commons license, unless indicated otherwise in a credit line to the material. If material is not included in the chapter's Creative Commons license and your intended use is not permitted by statutory regulation or exceeds the permitted use, you will need to obtain permission directly from the copyright holder. 